Démarche empirico, inductive de fouille de 86 mémoires déposés auprès de la Commission sur la démocratie canadienne et la responsabilisation des entreprises

\title{
LES REPRÉSENTATIONS DE 86 ACTEURS ÉCONOMIQUES FRANCOPHONES
}

\section{Aux confluents des discours sur la RSE au Canada}

Haykel Najlaoui, Emmanuelle Champion, Sophie Lévesque et Corinne Gendron

ESKA | « Revue internationale de psychosociologie »

2010/38 Vol. XVI | pages 99 à 117

ISSN 1260-1705

Article disponible en ligne à l'adresse :

https://www.cairn.inforevue-internationale-de-psychosociologie-2010-38-page-99.htm

Distribution électronique Cairn.info pour ESKA.

(C) ESKA. Tous droits réservés pour tous pays.

La reproduction ou représentation de cet article, notamment par photocopie, n'est autorisée que dans les limites des conditions générales d'utilisation du site ou, le cas échéant, des conditions générales de la licence souscrite par votre établissement. Toute autre reproduction ou représentation, en tout ou partie, sous quelque forme et de quelque manière que ce soit, est interdite sauf accord préalable et écrit de l'éditeur, en dehors des cas prévus par la législation en vigueur en France. Il est précisé que son stockage dans une base de données est également interdit. 
Démarche empirico inductive de fouille de 86 mémoires déposés auprès de la Commission sur la démocratie canadienne et la responsabilisation des entreprises

\section{LES REPRESENTATIONS DE 86 ACTEURS ECONOMIQUES FRANCOPHONES}

\section{Aux confluents des discours sur la RSE au Canada}
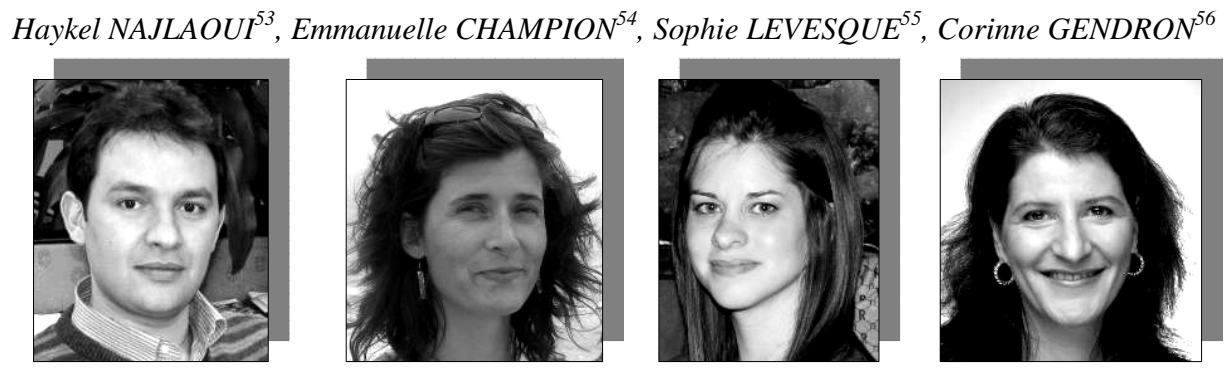

Au cours des dernières années, la responsabilité sociale de l'entreprise (RSE) a fait l'objet de nombreux débats au Canada. Alors que le rapport du comité mixte sur la gouvernance d'entreprise publié en 2001, plus connu sous le nom du rapport Saucier, insistait sur l'importance de parvenir à un équilibre des pouvoirs au sein de la gouvernance de l'entreprise, les multiples scandales financiers survenus principalement aux États Unis (Enron, Worldcom, etc.) ont rouvert le débat sur la nécessité d'une séparation claire des responsabilités des fiduciaires et des gestionnaires. Pour plusieurs, l'instauration de régimes d'options d'achat a conduit les dirigeants d'entreprise à travailler contre les intérêts des actionnaires si bien que des amendements à la Loi des sociétés par action ont été réclamés par des investisseurs institutionnels canadiens, notamment le Régime de retraite des enseignantes et des enseignants de l'Ontario (Lamoureux, 2002).

Étudiant à la maîtrise en sciences de l'environnement, Chaire de responsabilité sociale et de développement durable École des Sciences de la Gestion, Université du Québec à Montréal, Case postale 8888, succursale Centre-ville, Montréal (Québec) H3C 3P8 Canada, haykel.najlaoui@ @ourrier.uqam.ca Doctorante en Administration, Chaire de responsabilité sociale et de développement durable et membre $\mathrm{du}$ Centre de recherche interuniversitaire sur la mondialisation et le travail (CRIMT), champion.emmanuelle@uqam.ca Étudiante au Doctorat en Administration, Chaire de responsabilité sociale et de développement durable, levesque.sophie.4@courrier.uqam.ca

Titulaire, Chaire de responsabilité sociale et de développement durable, corinne.gendron@uqam.ca 
C'est dans ce contexte que la Commission sur la démocratie canadienne et la responsabilisation des entreprises a été lancée en 2001 afin de parvenir à une série de recommandations à l'intention du gouvernement canadien, pour mettre fin aux comportements délictueux des dirigeants d'entreprises. Financée par des fonds privés et totalement indépendante du gouvernement, cette Commission a connu un franc succès puisqu'elle a permis de colliger les recommandations, sous la forme de présentations et de mémoires, de plus de cent cinquante acteurs sociaux à travers le Canada. Dans un document de travail rédigé pour les audiences publiques, cette Commission proposait que le gouvernement canadien établisse un cadre réglementaire à partir de mécanismes existants dans le domaine de la RSE. Les différents acteurs ayant participé à ce débat ont pu enrichir les orientations proposées dans ce rapport.

Basée sur les mémoires déposés dans le cadre de cette Commission, l'analyse que nous proposons dans cet article vise à dégager des idéaux-types de la responsabilité sociale en vue de clarifier les débats qui ont émergé au cours des dernières années au Canada au sujet de la régulation des acteurs et des institutions économiques. Nous posons l'hypothèse que derrière le consensus apparent autour d'une nécessaire responsabilisation sociale des agents économiques, la pluralité des définitions de la responsabilité sociale est révélatrice des conflits sociaux autour du rôle de l'entreprise dans la société, de sa gouvernance, et du modèle de développement auquel elle concoure. Cependant, l'analyse des discours des entreprises, du patronat, des syndicats et des ONG que nous effectuons ici, permet d'identifier plusieurs points de rapprochement entre les représentations de ces acteurs, si bien qu'un éventuel consensus pourrait se consolider à l'avenir, en particulier sur les moyens à mettre en œuvre pour une régulation effective de la RSE.

\section{UNE APPROCHE SOCIOLOGIQUE À LA RSE}

Nous avons opté pour une approche sociologique, car bien que riche et abondante, la littérature managériale, à l'instar de l'approche gestionnaire dans son ensemble, pose problème pour appréhender le débat sur la RSE. Parfois même, elle tend à l'occulter en proposant un cadre ou une définition susceptibles de servir de référence qui se situent d'entrée de jeu dans une perspective managériale (Sethi, 1975 ; Wood, 1991 ; Epstein, 1987). Les études managériales sur la responsabilité sociale se limitent généralement à analyser les déterminants des comportements responsables ou avant-gardistes des agents ou des firmes. Par ailleurs, que ce soit sur la base d'études empiriques ou de réflexions théoriques, les auteurs s'entendent généralement sur le fait que le contenu et les contours de la responsabilité sociale corporative sont flous. Or, plutôt que de se pencher sur cette indétermination qui s'avère riche d'un point de vue social, ils se sont le plus souvent efforcés de réconcilier les différences en proposant une définition qui soit la «bonne» définition, ou encore en élaborant un cadre qui se veuille synthétique et intégrateur (Gendron, 2000). 
Il nous paraît donc opportun de mobiliser des cadres théoriques mieux adaptés pour saisir ce débat et analyser la pluralité des discours sur la responsabilité sociale au lieu de chercher à la dépasser, précisément parce que cette diversité est riche de sens sur le plan social. Or, parce qu'elle s'intéresse aux acteurs et aux conflits sociaux, mais aussi aux principes et aux modes de régulation sociale, la perspective sociologique nous semble toute désignée pour développer une analyse qui ne s'inscrive de prime abord ni dans une perspective fonctionnaliste, ni dans une approche exclusivement critique qui pourrait se révéler stérile. La perspective sociologique que nous proposons dans cet article déplace la question de la responsabilité sociale telle qu'elle est souvent abordée pour l'envisager moins comme un enjeu corporatif que comme une question de régulation sociale faisant intervenir, derrière l'institution que constitue l'entreprise, des acteurs sociaux en interaction.

L'École des représentations sociales avance que la pluralité des représentations sociales s'explique non seulement en fonction de l'information et de la pratique, mais aussi en fonction de la position sociale des acteurs sociaux : la représentation sociale porte la marque du sujet qui reconstruit l'objet et l'interprète tout en s'exprimant à travers elle (Jodelet, 1989). Bref, elle est sujette à un processus de focalisation qui répond à l'intérêt des sujets, tout en étant élaborée à partir de leur expérience. Les représentations de la responsabilité sociale que porte l'élite économique, tout comme celles des mouvements sociaux, sont donc fortement conditionnées par leur position d'élite ou d'acteur contestataire, leur expérience et leurs visées: par conséquent, elles ne sont pas neutres ou «objectives» et constituent un terrain de choix pour comprendre la forme et la teneur des clivages sociaux. Par ailleurs, compte tenu de leur position d'acteurs dominants, les représentations de l'élite économique tendront à s'imposer davantage que d'autres à l'ensemble de la société et deviendront agissantes sur l'organisation sociale et les avenues de modernisation sociale des institutions économiques.

Ainsi, les définitions de la responsabilité sociale diffèrent d'un acteur social à l'autre non seulement en raison d'une confusion ou d'une mécompréhension conceptuelle comme on le lit souvent, mais bien parce qu'à l'instar du progrès industriel à l'époque du fordisme ou du développement durable actuellement, la responsabilité sociale corporative traduit un idéal que peuvent revendiquer côte à côte des acteurs sociaux pourtant fondamentalement en désaccord sur le contenu, la forme et la mise en œuvre de cette responsabilité sociale. Il importe donc de rendre explicites les éléments de conflit concernant le rôle des entreprises dans la société et les modes de gouvernance à privilégier, mais aussi les nouveaux compromis sociaux qui pourraient se faire jour quant à ces questions. Dans la mesure où la multiplicité des définitions de la responsabilité sociale transpose les conflits sociaux autour de questions fondamentales telles que l'autonomie de l'élite économique, de la gouvernance, des processus de développement, de la participation aux institutions économiques et quant au partage de la richesse dans les sociétés modernes avancées, 
nous nous sommes demandés: quelles sont les définitions idéal-types de la responsabilité sociale pour chacun des acteurs sociaux (notamment les acteurs politiques, les acteurs économiques et les acteurs de la société civile) ? Sur quels éléments se cristallisent les conflits autour de la définition de la RSE ? Quels modèles de régulation peut-on envisager en regard de ces différentes représentations sociales de la responsabilité sociale corporative ? Peut-on identifier de nouveaux compromis sociaux post-fordistes relativement au rôle de l'entreprise dans la société ?

\section{MÉTHODOLOGIE}

Pour répondre à ces interrogations, nous nous sommes penchés sur les mémoires déposés dans le cadre de la Commission sur la démocratie canadienne et la responsabilisation des entreprises qui visait à collecter les points de vue des différents acteurs sur la RSE. Cette Commission nous a offert un terrain de choix pour réaliser cette recherche, puisque 157 mémoires touchant l'imputabilité des entreprises y ont été déposés par différents acteurs sociaux dans le cadre d'audiences publiques organisées dans plusieurs grandes villes canadiennes. Ces mémoires s'avèrent un excellent terrain pour dégager les représentations sociales que les différents acteurs sociaux ont de la RSE et surtout, des moyens à adopter pour sa mise en œuvre. Sur les 157 mémoires déposés, nous avons pu récupérer 86 mémoires auprès du secrétariat de la Commission.

Notre catégorisation révèle pas moins de dix acteurs au sein de ce corpus, ce qui offre un éventail de représentations sociales important et varié, soit celles de l'acteur «entreprise» $(\mathrm{n}=4)$, de l'acteur «association patronale» $(\mathrm{n}=4)$, de l'acteur « organisation non gouvernementale » $(\mathrm{n}=17)$, de l'acteur « finance responsable » $(\mathrm{n}=9)$, de l'acteur «État » $(\mathrm{n}=4)$, de l'acteur « organisation religieuse » $(\mathrm{n}=3)$, de l'acteur syndicat» $(\mathrm{n}=9)$, de l'acteur «éducation» $(\mathrm{n}=1)$, de l'acteur «chercheur» $(\mathrm{n}=8)$ et de l'acteur « citoyen » $(\mathrm{n}=15)$. Pour les fins de cet article, nous concentrerons notre analyse sur quatre acteurs seulement (Tableau 1), c'est-àdire les entreprises, le patronat, les ONG et les syndicats. Cet échantillon nous permettra néanmoins de déterminer les représentations sociales qui se font actuellement concurrence entre les acteurs les plus influents quant à la définition de la RSE, le rôle de l'entreprise, la gouvernance de l'entreprise et le modèle de développement qui concoure à la responsabilisation des entreprises. 
Tableau 1 - Présentation des acteurs et des mémoires analysés

\begin{tabular}{|c|c|c|c|c|}
\hline & $\begin{array}{c}\text { Nom de } \\
\text { l'organisation }\end{array}$ & Titre du mémoire & $\begin{array}{l}\text { Nbre de } \\
\text { pages }\end{array}$ & $\begin{array}{l}\text { Nbre de } \\
\text { citations }\end{array}$ \\
\hline \multirow{4}{*}{ 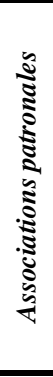 } & $\begin{array}{l}\text { Conference Board of } \\
\text { Canada }\end{array}$ & $\begin{array}{l}\text { Presentation to the Commissioners } \\
\text { Canadian Democracy and Corporate } \\
\text { Accountability project }\end{array}$ & 5 & 18 \\
\hline & $\begin{array}{l}\text { Canadian Centre for } \\
\text { Ethics and corporate } \\
\text { policy }\end{array}$ & $\begin{array}{llc}\text { Submission to the } & \text { Canadian } \\
\text { democracy } & \& & \text { corporate } \\
\text { accountability } & \text { commission } & \\
\end{array}$ & 5 & 24 \\
\hline & $\begin{array}{ll}\text { Jeune Chambre } & \mathrm{du} \\
\text { Commerce } & \mathrm{de} \\
\text { Montréal } & \\
\end{array}$ & $\begin{array}{l}\text { Mémoire sur la Responsabilité sociale } \\
\text { de l'entreprise }\end{array}$ & 17 & 42 \\
\hline & $\begin{array}{l}\text { Business Council on } \\
\text { national issues }\end{array}$ & $\begin{array}{l}\text { Globalisation, citizenship } \\
\text { competitiveness. }\end{array}$ & 13 & 44 \\
\hline \multirow{4}{*}{ 文 } & $\begin{array}{l}\text { Grainger } \\
\text { Associates }\end{array}$ & $\begin{array}{l}\text { Canadian Democracy and Corporate } \\
\text { Accountability Commission }\end{array}$ & 17 & 12 \\
\hline & Shell Canada & Shell Canada Limited Response & 4 & 17 \\
\hline & Talisman Energy & $\begin{array}{l}\text { Chairmen and Commissioners } \\
\text { Canadian Democracy and Corporate } \\
\text { Accountability Commission }\end{array}$ & 5 & 10 \\
\hline & Suncor Energy & $\begin{array}{l}\text { Suncor Energy Corporate Social } \\
\text { Responsibility Policy DRAFT - June } \\
2001\end{array}$ & 13 & 21 \\
\hline \multirow{6}{*}{ 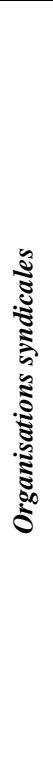 } & $\begin{array}{l}\text { United Steelworkers } \\
\text { of America }\end{array}$ & $\begin{array}{l}\text { Corporate accountability and the } \\
\text { right to unionize : two Canadian case } \\
\text { studies by Lawrence McBearty, } \\
\text { National director for Canada }\end{array}$ & 15 & 61 \\
\hline & $\begin{array}{lr}\text { Fédération } & \text { des } \\
\text { travailleurs et } & \begin{array}{r}\text { des } \\
\text { travailleuses }\end{array} \\
\text { Québec (FTQ) } & \\
\end{array}$ & $\begin{array}{l}\text { Mémoire de la Fédération des } \\
\text { travailleurs et des travailleuses du } \\
\text { Québec }\end{array}$ & 14 & 44 \\
\hline & $\begin{array}{l}\text { Canadian Labour } \\
\text { Congress (CLC) }\end{array}$ & $\begin{array}{l}\text { Presentation to the Canadian } \\
\text { Democracy and Corporate } \\
\text { Accountability Commission by Nancy } \\
\text { Riche, Secretary Treasurer }\end{array}$ & 10 & 51 \\
\hline & $\begin{array}{l}\text { BC } \\
\text { Labour }\end{array}$ & $\begin{array}{l}\text { Presentation to the Canadian } \\
\text { democracy and corporate } \\
\text { accountability commission by Jim } \\
\text { Sinclair, President }\end{array}$ & 9 & 37 \\
\hline & $\begin{array}{l}\text { Syndicat des } \\
\text { communications, des } \\
\text { énergies et du papier } \\
(\text { SCEP) }\end{array}$ & $\begin{array}{l}\text { Syndicat des communications, des } \\
\text { énergies et du papier, Peter Murdoch }\end{array}$ & 4 & 26 \\
\hline & $\begin{array}{l}\text { Canadian } \\
\text { Automobile Workers }\end{array}$ & $\begin{array}{l}\text { Speaking notes }- \text { Presentation to } \\
\text { Corporate Accountability by Buzz } \\
\text { Hargrove, President }\end{array}$ & 9 & 57 \\
\hline
\end{tabular}




\begin{tabular}{|c|c|c|c|c|}
\hline & $\begin{array}{l}\text { Calgary and District } \\
\text { labour council }\end{array}$ & $\begin{array}{l}\text { Submission to the Canadian } \\
\text { Democracy and } \text { Corporate } \\
\text { Accountability Commission by Estelle } \\
\text { Kuzyk, 2nd Vice President }\end{array}$ & 2 & 10 \\
\hline & CUPE Manitoba & $\begin{array}{l}\text { Presentation to the democracy and } \\
\text { corporate accountability commission } \\
\text { CUPE Manitoba by Paul Moist, } \\
\text { President CUPE, Manitoba }\end{array}$ & 15 & 61 \\
\hline & $\begin{array}{l}\text { Manitoba Federation } \\
\text { of Labour }\end{array}$ & $\begin{array}{l}\text { Brief to the democracy and } \\
\text { accountability commission }\end{array}$ & 10 & 53 \\
\hline \multirow{10}{*}{ 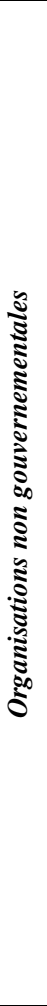 } & Halifax Initiative & $\begin{array}{l}\text { Submission to the } \begin{array}{l}\text { Canadian } \\
\text { democracy and } \\
\text { accountability } \\
\text { commission }\end{array} \\
\end{array}$ & 4 & 16 \\
\hline & $\begin{array}{l}\text { Canadians Friends of } \\
\text { Burma }\end{array}$ & $\begin{array}{l}\text { Canadian Corporate Accountability } \\
\text { and Burma }\end{array}$ & 5 & 23 \\
\hline & $\begin{array}{l}\text { Amnesty } \\
\text { International }\end{array}$ & $\begin{array}{l}\text { Submission to the } \begin{array}{l}\text { Canadian } \\
\text { democracy and }\end{array} \\
\text { accountability commission }\end{array}$ & 2 & 7 \\
\hline & $\begin{array}{l}\text { Conservation Council } \\
\text { of Ontario }\end{array}$ & $\begin{array}{l}\text { Corporate Accountability and the } \\
\text { Environment }\end{array}$ & 4 & 28 \\
\hline & $\begin{array}{l}\text { Canadian Council for } \\
\text { International } \\
\text { Cooperation }\end{array}$ & $\begin{array}{l}\text { Submission to the } \begin{array}{l}\text { Canadian } \\
\text { democracy and }\end{array} \\
\text { corporate } \\
\text { accountability commission }\end{array}$ & 10 & 39 \\
\hline & $\begin{array}{l}\text { Defense of Canadian } \\
\text { Liberty Committee }\end{array}$ & $\begin{array}{l}\text { Canadian Democracy \& Corporate } \\
\text { Accountability }\end{array}$ & 4 & 22 \\
\hline & Aurora Institute & $\begin{array}{lll}\text { Submission to the } & \text { Canadian } \\
\text { democracy and } & \text { corporate } \\
\text { accountability commission } & \\
\end{array}$ & 17 & 99 \\
\hline & $\begin{array}{lr}\text { Anti-poverty } & \text { groups } \\
\text { of British } & \text { Columbia } \\
\text { and the } & \text { British } \\
\text { Columbia } & \text { Public } \\
\text { Interest } & \text { Advocacy } \\
\text { Centre } & \end{array}$ & $\begin{array}{l}\text { Corporate accountability and social } \\
\text { responsibility }\end{array}$ & 6 & 31 \\
\hline & $\begin{array}{l}\text { Ethical Trading } \\
\text { Action Group }\end{array}$ & $\begin{array}{l}\text { Submission to the } \begin{array}{l}\text { Canadian } \\
\text { democracy and } \\
\text { accountability } \\
\text { commission }\end{array} \\
\end{array}$ & 6 & 30 \\
\hline & Democracy Watch & $\begin{array}{l}\text { Opening statement to the Canadian } \\
\text { Democracy and Corporate } \\
\text { Accountability Commission }\end{array}$ & 15 & 78 \\
\hline
\end{tabular}

Nous avons étudié l'ensemble des mémoires présentés ci-dessus en vue de réaliser une analyse de contenu qui nous a permis de dégager les éléments de construction sociale concernant la RSE et son imputabilité. Afin de mettre à profit une perspective de théorisation ancrée, l'utilisation du logiciel Atlas.ti a donné lieu notamment à la réalisation d'une cartographie perceptuelle des éléments de responsabilité sociale corporative. En ce qui concerne les étapes suivies, après avoir répertorié les mémoires et effectué les regroupements par catégorie d'acteurs, nous 
avons procédé, dans un premier temps, à une codification ouverte, puis axiale de chaque mémoire pour faire ressortir la logique discursive particulière (mots clefs, vocabulaire, objectifs du mémoire, arguments principaux et lecture du contexte canadien et international). Ceci nous a permis de caractériser chaque mémoire en fonction de l'organisme dont il est issu. Dans un deuxième temps, nous avons exécuté une codification axiale de deuxième niveau appliquée au regroupement des mémoires par catégorie d'acteurs. Ce faisant, nous avons tenté de cerner non seulement les points communs, mais aussi les éventuelles discordances entre les conceptions de la responsabilité sociale chez des organisations représentatives d'une même catégorie d'acteur social. Enfin, dans un troisième temps, nous avons regroupé au sein d'une unité herméneutique commune l'ensemble des mémoires des différents acteurs en vue de saisir les ruptures conceptuelles concernant la RSE, ainsi que les éléments de problématique connexes tels que la régulation et l'autonomie des acteurs économiques, la légitimité des interventions politiques, le rôle de l'entreprise dans la société, ses modalités de gouvernance et enfin le modèle de développement auquel il est souhaitable qu'elle contribue.

\section{RÉSULTATS}

Nous allons, dans un premier temps, présenter les codes les plus fréquents révélateurs des thèmes de prédilection de chacun de nos acteurs ainsi que les codes qui présentent une certaine pertinence pour notre questionnement de recherche. Ces résultats seront présentés par catégorie d'acteurs, soit les associations patronales, les entreprises, les syndicats et les ONG. Dans un deuxième temps, nous discuterons des représentations de la RSE des différents acteurs afin d'identifier des idéaux types pour mettre ceux-ci en dialogue.

\section{Les associations patronales}

Le discours des associations patronales met en évidence la nécessité de reconnaître, d'une part, les impacts sociaux et environnementaux des activités corporatives et d'autre part, la RSE. Cette prise en compte s'opère principalement par le biais de la reddition de comptes ou plus précisément de la divulgation extra financière qui vise à informer les Parties Prenantes de l'entreprise. Les associations patronales associent la RSE à un comportement moral par rapport à toutes les Parties Prenantes de l'entreprise sans toutefois le définir. Leur discours évoque plusieurs Parties Prenantes, à savoir les communautés $(n=22)$, les gouvernements $(n=21)$, les employés $(n=19)$, les actionnaires $(n=18)$ et les clients $(n=12)$. 
Tableau 2 - Les codes les plus fréquents et les codes pertinents dans le discours des associations patronales

\begin{tabular}{|c|c|c|c|}
\hline Codes & 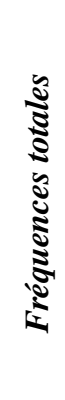 & 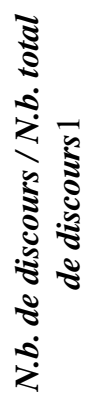 & 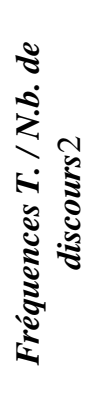 \\
\hline 1. Responsabilité sociale de l'entreprise & 76 & $4 / 4$ & 15 \\
\hline 2. Parties prenantes & 23 & $3 / 4$ & 8 \\
\hline 3. Réglementation & 23 & $5 / 4$ & 7 \\
\hline 4. Communautés & 22 & $4 / 4$ & 4 \\
\hline 5. Reddition de comptes / Bilan RSE & 22 & $3 / 4$ & 7 \\
\hline 6. Gouvernements & 21 & $4 / 4$ & 5 \\
\hline 7. Employés & 19 & $4 / 4$ & 4 \\
\hline 8. Actionnaires & 18 & $4 / 4$ & 4 \\
\hline 9. Rentabilité de l'entreprise & 18 & $4 / 4$ & 4 \\
\hline 10. Normes et standards & 14 & $4 / 4$ & 4 \\
\hline 11. Gestionnaires & 14 & $4 / 4$ & 4 \\
\hline 12. Clients & 12 & $4 / 4$ & 3 \\
\hline 13. Image de l'entreprise & 10 & $3 / 4$ & 3 \\
\hline 14. Environnement & 3 & $3 / 4$ & 1 \\
\hline
\end{tabular}

Dans la perspective patronale, l'idée dominante est d'assurer la rentabilité en intégrant sur une base volontaire les intérêts des Parties Prenantes aux décisions de l'entreprise. Cette intégration est censée générer des pratiques socialement responsables rentables pour l'entreprise. À l'interne, la rentabilité de la RSE passe par de bonnes relations avec les employés, ce qui ultimement favorise l'attractivité auprès des candidats potentiels et le maintien des compétences cruciales à la compétitivité. À l'externe, les bonnes relations avec les Parties Prenantes procurent une image corporative positive et un avantage concurrentiel auprès des investisseurs, une plus grande acceptation ou intégration auprès des communautés et un pouvoir de négociation face au gouvernement. En ce sens, les discours des associations patronales vont jusqu'à faire de la RSE une occasion d'affaires. 
Les associations patronales présentent la reddition de comptes $(n=22)$ comme premier déterminant de la RSE. La reddition de comptes est d'un côté une pratique responsable de l'entreprise et de l'autre côté, un outil qui permet de mesurer la performance de l'entreprise en regard des attentes des Parties Prenantes. Toutefois, elle permet de promouvoir le comportement socialement responsable qu'à la condition d'être (1) volontaire et (2) encadrée par une norme de procédures.

La conformité légale ne peut circonscrire le concept de la RSE, qui est par essence volontaire, ouvert à différentes initiatives et dont la nature est précisément d'aller au-delà de la conformité réglementaire. Cette position est néanmoins mitigée. Pour le Business Council on National Issues et la Jeune Chambre de Commerce de Montréal, la réglementation est le moyen par lequel le gouvernement contrôle le comportement des acteurs économiques. Elle a souvent des conséquences graves non seulement sur la compétitivité des entreprises canadiennes, mais également sur le système démocratique. Par contre, les deux autres associations patronales, le Canadian Centre for Ethics \& Corporate Policy et le Conference Board of Canada laissent plus de place à la réglementation. En effet, après avoir défendu la thèse selon laquelle la RSE est volontaire, ces deux associations patronales recommandent l'amendement de la Loi canadienne sur les sociétés par actions pour permettre aux gestionnaires d'intégrer légalement les intérêts de ces Parties Prenantes nonactionnaires à leur prise de décision. Cette proposition d'amendement n'arrête en aucun cas le contenu de la RSE, elle laisse en effet la voie libre pour le développement d'initiatives volontaires.

\section{Les entreprises}

Dans leur discours, les entreprises ne définissent pas la RSE, mais elles évoquent des notions et des concepts tels que le développement durable $(\mathrm{n}=8)$, l'éthique corporative $(n=8)$, les valeurs de l'entreprise $(n=7)$ et les générations futures $(n=2)$. Aussi, elles font un tour d'horizon des principes qui régissent la conduite de leurs affaires et les actions qui en découlent. Les employés $(n=19)$ et les communautés $(\mathrm{n}=17)$ sont les Parties Prenantes les plus fréquentes dans les discours et par rapport auxquelles les entreprises identifient une panoplie de pratiques responsables comme la consultation et la concertation, la conformité aux lois et aux normes et aux standards, la reddition de comptes et la préservation de l'environnement. 
Tableau 3 - Les codes les plus fréquents et les codes pertinents dans le discours des entreprises

\begin{tabular}{|c|c|c|c|c|}
\hline & Codes & 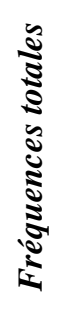 & 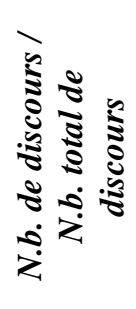 & 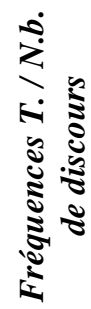 \\
\hline 1. & Responsabilité sociale de l'entreprise & 28 & $4 / 4$ & 7 \\
\hline 2. & Employés & 19 & $3 / 4$ & 7 \\
\hline 3. & Communautés & 17 & $3 / 4$ & 6 \\
\hline 4. & Parties Prenantes & 13 & $4 / 4$ & 3 \\
\hline 5. & Actionnaires & $\mathbf{1 0}$ & $3 / 4$ & 3 \\
\hline 6. & Gouvernement & 8 & $3 / 4$ & 3 \\
\hline 7. & Développement durable & 8 & $3 / 4$ & 3 \\
\hline 8. & Environnement & 8 & $4 / 4$ & 2 \\
\hline 9. & Consultation et concertation & 8 & $3 / 4$ & 3 \\
\hline 10. & Éthique corporative & 8 & $2 / 4$ & 4 \\
\hline 11. & Transparence & 7 & $4 / 4$ & 2 \\
\hline 12. & Valeurs de l'entreprise & 7 & $3 / 4$ & 2 \\
\hline 13. & Normes et standards & 6 & $3 / 4$ & 2 \\
\hline & Reddition de comptes & 6 & $3 / 4$ & 2 \\
\hline 15. & Générations futures & 2 & $2 / 4$ & 1 \\
\hline
\end{tabular}

L'idée principale soutenue dans tous les mémoires des entreprises est que leur responsabilité ne se limite pas à garantir un retour sur l'investissement pour les actionnaires. Il s'agit également de créer de la richesse pour les employés et les communautés et de travailler en étroite collaboration avec toutes les Parties Prenantes. Toutefois, les mémoires des entreprises font ressortir deux visions de la RSE. Dans la première vision, Suncor Energy et Grainger and Associates précisent que la conduite des affaires doit se faire d'une façon éthique. Elles parlent ainsi de l'éthique corporative ou de la conduite éthique des affaires définies comme étant une gestion qui se base sur un ensemble de principes et de valeurs tels que l'intégrité, la transparence et le respect des droits humains, de l'environnement et des normes internationales en matière de santé et sécurité au travail. La conduite éthique des affaires inclut également la concurrence loyale, la conformité aux lois et aux règlements et la gestion environnementale.

Dans la deuxième vision, Shell Canada et Talisman Energy, font plutôt référence dans leur discours portant sur la RSE au développement durable et à la création

Revue Internationale de Psychosociologie, année 2010 
«durable» des richesses. Shell, par exemple, définit le développement durable comme étant le continuum de trois dimensions : l'économie, l'environnement et le social. Quant à la responsabilité sociale de l'entreprise, elle constitue un engagement par lequel l'entreprise reconnaît sa responsabilité de consulter, de communiquer et de rendre compte à ses Parties Prenantes, notamment les communautés et les employés. Ces éléments rejoignent la vision de Talisman Energy, qui s'appuie sur la reddition de comptes et la vérification indépendante pour produire des richesses d'une façon durable tout en préservant les générations futures. Dans cette vision, l'environnement est souvent évoqué pour affirmer un leadership. Shell Canada et Talisman Energy reconnaissent leur responsabilité sociale en la matière, particulièrement celles ayant trait à l'exploitation et la transformation des ressources naturelles ainsi que celles concernant la commercialisation des produits et services énergétiques.

Dans leurs mémoires, la responsabilité de l'entreprise envers les employés consiste à offrir un milieu de travail tolérant, ouvert à toute diversité et respectueux des droits humains. Quant à leurs responsabilités envers les autres Parties Prenantes et plus particulièrement envers les communautés, les entreprises identifient la consultation en tant que première pratique de la RSE et qui vise l'intégration de leurs intérêts. La deuxième pratique mise de l'avant est la reddition de comptes qui, par le biais d'une vérification indépendante, garantit aux Parties Prenantes la prise en compte de leurs recommandations.

\section{Les syndicats}

Le discours des syndicats est axé sur les lois et les règlements $(\mathrm{n}=95)$, en faisant plus précisément référence au droit du travail $(\mathrm{n}=53)$ et au droit d'association $(n=40)$, mais il inclut également, lorsqu'il est question du renforcement de la RSE, des références à des mécanismes juridiquement non contraignants tels que les codes de conduite $(n=12)$, les sanctions économiques $(n=15)$ et la finance socialement responsable $(\mathrm{n}=17)$. Il est intéressant de remarquer que seul le syndicat Congrès $d u$ travail du Canada (CTC-CLC) évoquait les accords-cadres internationaux $(\mathrm{n}=1)$ dans son mémoire. Ces mécanismes devraient néanmoins s'accompagner de mesures spécifiques pour veiller à leur conformité $(n=31)$. En effet, la question de l'imputabilité des entreprises $(\mathrm{n}=53)$, qui se pose à la fois pour l'application du droit $(n=13)$ et des mécanismes volontaires $(n=6)$, était une des préoccupations exprimées par l'acteur syndical. 
Tableau 4 - Les codes les plus fréquents et les pertinents dans le discours des syndicats

\begin{tabular}{|c|c|c|c|}
\hline Codes & 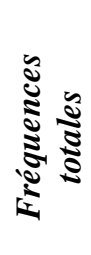 & 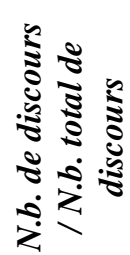 & 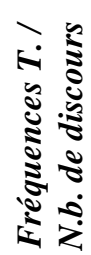 \\
\hline 1. Travailleurs & 98 & $9 / 9$ & 11 \\
\hline 2. Lois et règlements & 95 & $8 / 9$ & 12 \\
\hline 3. Gouvernement & 90 & $9 / 9$ & 10 \\
\hline 4. Droit du travail & 58 & $7 / 9$ & 8.5 \\
\hline 5. Amendement-Reforme & 44 & $5 / 9$ & 9 \\
\hline 6. Droit d'association & 40 & $5 / 9$ & 8 \\
\hline 7. Stratégies antisyndicales & 41 & $5 / 9$ & 8 \\
\hline 8. Mesures de conformité & 31 & $8 / 9$ & 4 \\
\hline 9. Innovation & 31 & $5 / 9$ & 6 \\
\hline 10. Codes de conduite & 12 & $5 / 9$ & 2.4 \\
\hline 11. Transparence & 20 & $8 / 9$ & 2.5 \\
\hline 12. Investissement responsable & 17 & $5 / 9$ & 3.4 \\
\hline 13. Parties Prenantes & 17 & $7 / 9$ & 2.5 \\
\hline 14. Environnement & 17 & $7 / 9$ & 2.5 \\
\hline 15. Reddition de comptes & 13 & $5 / 9$ & 2.6 \\
\hline
\end{tabular}

Dans leurs mémoires, les organisations syndicales font état d'un recul du droit du travail $(\mathrm{n}=53)$ au Canada. L'érosion du droit du travail est associée à la perte de pouvoir des organisations syndicales du fait de stratégies antisyndicales $(n=41)$, mises en œuvre par plusieurs directions d'entreprise, ce qui nuit par conséquent au droit d'association $(n=40)$. Certains syndicats décrivent en détails les intimidations dont font l'objet les travailleurs $(n=8)$ et les licenciements dont ils sont victimes $(\mathrm{n}=9)$ alors que ces employés cherchent à se faire représenter par une organisation syndicale.

Lorsqu'il est question de lois et de règlements $(n=95)$, les organisations syndicales insistent sur la nécessité d'amender certaines lois pour parvenir à un encadrement effectif des entreprises transnationales. À ce chapitre, les syndicats s'entendent sur l'idée de conditionner l'octroi de contrats publics en fonction de la performance sociale et environnementale des entreprises. Ils appuient également la recommandation à l'effet que le droit corporatif soit amendé en vue de clarifier les possibilités pour les actionnaires et les fiduciaires de déposer des résolutions à caractère social.

Aussi, la dimension du pouvoir $(n=62)$ est très présente dans le discours des syndicats surtout lorsqu'il est question des entreprises privées $(n=45)$. Pour les

Revue Internationale de Psychosociologie, année 2010 
syndicats, la mondialisation des économies $(\mathrm{n}=39)$ et son lot de mesures visant la libéralisation du commerce $(n=24)$ ont contribué à émanciper les entreprises des rapports de force industriels. Dans le but d'inscrire leur mobilisation $(\mathrm{n}=32)$ au sein d'une économie mondialisée et ainsi faire contrepoids aux entreprises transnationales, les syndicats se prononcent en faveur d'un cadre réglementaire régulatoire international en matière de RSE au sein duquel ils seraient les garants des droits des travailleurs $(n=98)$. En outre, ce système régulatoire s'appuierait sur différents instruments visant à conférer un certain degré de transparence $(\mathrm{n}=20)$ sur la gestion de l'entreprise. Comme le proposait initialement la Commission Broadbent, il pourrait s'agir de rendre obligatoire la publication d'un bilan social ou d'un rapport de performance extra financière $(\mathrm{n}=13)$ en amendant la Loi sur les sociétés par actions.

\section{Les organisations non gouvernementales}

Les mémoires des organisations non gouvernementales portent leur attention principalement sur les lois et les règlements $(n=109)$. La société $(n=103)$, le gouvernement $(n=96)$, la responsabilité sociale des entreprises $(n=79)$, l'imputabilité $(n=61)$ et la reddition de comptes $(n=61)$ émergent également comme des sujets d'intérêts auprès des ONG. Enfin, une troisième série de codes se démarquent : l'environnement $(n=46)$, les droits humains $(n=45)$, les normes et standards $(n=43)$, les actionnaires $(n=35)$, les employés $(n=30)$, la communauté $(n=24)$, la rentabilité financière $(n=24)$ et les valeurs $(n=21)$.

Tableau 5. Les codes les plus fréquents et pertinents dans le discours des ONG

\begin{tabular}{|ll|c|c|c|}
\hline \multicolumn{1}{|c|}{ Codes } & Fréquences \\
totales & $\begin{array}{c}\text { N.b. de } \\
\text { discours / N.b. } \\
\text { total de } \\
\text { discours }\end{array}$ & $\begin{array}{c}\text { Fréquences T. / } \\
\text { N.b.de discours }\end{array}$ \\
\hline 1. & Loi et règlements & $\mathbf{1 0 9}$ & $9 / 10$ & 12 \\
\hline 2. & Société & $\mathbf{1 0 3}$ & $11 / 10$ & 9 \\
\hline 3. & Gouvernement & $\mathbf{9 6}$ & $10 / 10$ & 10 \\
\hline 4. & Responsabilité sociale & $\mathbf{7 9}$ & $11 / 10$ & 7 \\
\hline 5. & Imputabilité & $\mathbf{6 1}$ & $8 / 10$ & 8 \\
\hline 6. & Reddition de comptes & $\mathbf{6 1}$ & $10 / 10$ & 5 \\
\hline 7. & Environnement & $\mathbf{4 6}$ & $8 / 10$ & 6 \\
\hline 8. & Droits humains & $\mathbf{4 5}$ & $8 / 10$ & 6 \\
\hline 9. & Normes et standards & $\mathbf{4 3}$ & $8 / 10$ & 5 \\
\hline 10. & Actionnaires & $\mathbf{3 5}$ & $6 / 10$ & 6 \\
\hline 11. & Employés & $\mathbf{3 0}$ & $7 / 10$ & 4 \\
\hline 12. & ONG & $\mathbf{2 6}$ & $8 / 10$ & 3 \\
\hline 13. & Communautés & $\mathbf{2 4}$ & $6 / 10$ & 4 \\
\hline 14. & Rentabilité/Résultat financier & $\mathbf{2 4}$ & $7 / 10$ & 3 \\
\hline 15. & Valeurs & $\mathbf{2 1}$ & $7 / 10$ & 3 \\
\hline
\end{tabular}

De façon générale, les ONG considèrent que les mesures volontaires tels que les codes de conduite ou d'éthique $(n=20)$ mises de l'avant par les entreprises sont bien insuffisantes et ce, indépendamment du secteur d'activité. En effet, les ONG 
souhaitent voir des réformes administratives et institutionnelles obligeant les entreprises à améliorer leur performance sociale et environnementale. À cet effet, notons que les ONG estiment que cette approche de nature coercitive constitue un retour aux sources en regard du rôle du gouvernement. D'ailleurs, dans cet ordre d'idées, les ONG font front commun lorsqu'il s'agit de pointer l'incapacité du marché à réguler l'économie. Le gouvernement $(\mathrm{n}=96)$ doit jouer un rôle beaucoup plus substantiel dans la régulation de l'économie et par conséquent, il est tenu de formuler des normes, des standards $(n=43)$ ou un référentiel qui forcerait l'institutionnalisation de comportements responsables.

La définition de la RSE $(n=79)$ pour chaque ONG semble intimement liée à son champ d'intervention. Par exemple, pour le Canadian Council for International Cooperation, la RSE se définit comme le respect des droits humains, des normes du travail et de certaines normes environnementales par les entreprises canadiennes qui œuvrent à l'étranger alors que pour le Conservation Council of Ontario, la RSE se rapporte plutôt à la protection de la nature, à la conservation des ressources et à la prévention de la pollution. Cependant, malgré ces spécificités, deux dimensions propres à la RSE font l'objet d'une attention soutenue dans la plupart des mémoires, soit le respect de l'environnement $(n=46)$ et des droits humains $(n=45)$. Notons que de façon générale, le respect de l'environnement fait référence à la diminution de la pollution et à la protection des ressources non renouvelables tandis que les droits humains sont ceux répertoriés dans les déclarations internationales telle que la Déclaration des droits humains. Ainsi, l'imputabilité $(n=61)$ des entreprises est principalement associée aux actions ou gestes pouvant avoir des conséquences néfastes sur l'environnement ou les droits humains.

Pour les ONG, la reddition de comptes $(\mathrm{n}=61)$, principalement envers la société $(n=103)$ et le gouvernement $(n=96)$, mais aussi envers les actionnaires $(n=35)$, les employés $(n=30)$, la communauté $(n=24)$ et les consommateurs $(n=19)$ s'accompagne d'un bilan social ou d'autres outils qui obligent les entreprises à divulguer des informations extra financières.

\section{AUX CONFLUENTS DES DISCOURS SUR LA RSE AU CANADA}

Après avoir présenté les discours des acteurs, nous proposons à présent de faire une analyse par thème afin de dégager les représentations de la RSE, de la régulation et du rôle de l'entreprise et pour finir, du modèle de développement envisagé par les acteurs. Puisqu'il s'agissait également d'identifier des points de rapprochement entre les représentations de nos acteurs, nous avons décelé une convergence au sein de notre corpus : la reddition de comptes.

\section{Les représentations de la $\mathrm{RSE}$}

Plusieurs représentations de la RSE se dégagent du discours des acteurs analysés (Tableau 6). Les associations patronales et les entreprises présentent dans l'ensemble des représentations semblables en ce qui a trait à la nécessité d'une 
certaine responsabilisation des entreprises par rapport aux impacts sociaux et environnementaux et surtout, en faisant front commun contre la réglementation. Il s'agit d'une RSE volontaire conciliée à la rentabilité économique et qui se concrétise par la divulgation et la reddition de comptes. Sa mise en œuvre se résume à l'intégration des intérêts des Parties Prenantes par l'entremise de la consultation surtout lorsque la prise en compte des Parties Prenantes devient stratégique et rentable. À cet effet, notons que la RSE semble favoriser l'attrait auprès d'employés potentiels (Turban et Greening, 1997 ; Greening et Turban, 2000 ; Schmidt-Albinger et Freeman, 2000 ; Luce et al., 2001 ; Backhaus et al. 2002 ; Turban et Cable, 2003), l'amélioration du climat organisationnel (Morris, 1997), les comportements hors rôle (Luce, 1998 citée dans Corley et al., 2001) communément appelés les comportements discrétionnaires (Simard et Tremblay, 2000), la motivation (Turban et Greening, 1997 ; Greening et Turban, 2000) et le maintien des compétences cruciales à la compétitivité. Plus encore, il semble qu'une entreprise socialement responsable améliorerait son image ou sa réputation organisationnelle (Riordan et al. 1997) et obtiendrait plus facilement du financement (Teoh et Shiu, 1990 ; Epstein et al., 1994 ; Waddock et Graves, 1997 ; Stenzel et Stenzel, 2005). Toutefois, pour ce qui est d'une définition de la RSE, on distingue deux approches : le premier groupe associe la RSE à l'éthique des affaires alors que le deuxième la rattache au développement durable. Dans le dernier cas, les entreprises doivent viser la durabilité en ce qui concerne la création de richesses, non seulement pour les actionnaires, mais aussi pour les employés et les communautés.

Les représentations des associations syndicales et des ONG présentent pour leur part des traits communs sur la question du respect des droits humains, des normes du travail, des conventions internationales en matière de conservation de la nature et de prévention de la pollution. En termes de contenu, les représentations des associations syndicales et des ONG se basent sur la charte des droits humains ou les conventions internationales. Les deux représentations convergent également par rapport au rôle que devrait jouer l'entreprise à titre d'institution sociale. En effet, l'entreprise résulte de choix collectifs et se doit à ce titre de redistribuer les richesses créées collectivement, c'est-à-dire avec l'appui de la société et grâce au travail de ses employés. Ce point de vue va à l'encontre des représentations des entreprises et des associations patronales qui s'accrochent à la mission juridique de l'entreprise axée sur la création de richesse pour les actionnaires. Cette vision corporative du rôle de l'entreprise ne nie pas la contribution de l'entreprise au développement et au progrès social, mais l'envisage plutôt en tant qu'acteur économique.

Si l'on tente de définir un idéal type d'un éventuel cadre régulatoire par acteur, les entreprises et les associations patronales mettent de l'avant la thèse du volontarisme. Ce cadre n'exclut pas une certaine forme de réglementation qui donnera plus de pouvoir aux gestionnaires en leur permettant notamment d'intégrer les intérêts des Parties Prenantes. L'autorégulation défendue par les entreprises et les associations patronales n'est pas totalement rejetée par les organisations syndicales, puisqu'elles endossent la constitution d'un système hybride qui comprendrait à la 
fois des mesures coercitives, des mesures volontaires et même la possibilité d'utiliser les mécanismes de marché. Par contre, l'ensemble des ONG rejette les mesures volontaires et la régulation par le marché. Pour cet acteur, de nouvelles réformes administratives et institutionnelles s'imposent pour améliorer la performance sociale et environnementale des entreprises et assurer la régulation du système économique.

Tableau 6 - Les différentes conceptions de la RSE

\begin{tabular}{|c|c|c|c|c|}
\hline & $\begin{array}{c}\text { Associations } \\
\text { patronales }\end{array}$ & Entreprises & $\begin{array}{c}\begin{array}{c}\text { Organisations } \\
\text { syndicales }\end{array} \\
\end{array}$ & ONG \\
\hline 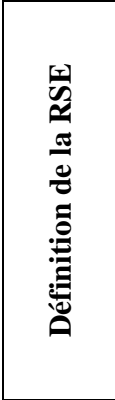 & $\begin{array}{l}\text { - Reconnaissance } \\
\text { des impacts sociaux } \\
\text { et } \\
\text { environnementaux } \\
\text { de l'entreprise } \\
\text { - RSE volontaire } \\
\text { - RSE va de pair } \\
\text { avec la rentabilité } \\
\text { économique }\end{array}$ & $\begin{array}{l}\text { - Deux groupes : } \\
\text { 1. RSE } \\
\text { synonyme } \\
\text { d'éthique des } \\
\text { affaires } \\
\text { 2. RSE ancrée } \\
\text { dans une vision } \\
\text { de } \\
\text { développement } \\
\text { durable }\end{array}$ & $\begin{array}{l}\text { - La RSE se } \\
\text { rapporte } \\
\text { principalement au } \\
\text { droit du travail et à } \\
\text { la protection de } \\
\text { l'environnement et } \\
\text { se base sur les } \\
\text { normes et les } \\
\text { conventions } \\
\text { internationales }\end{array}$ & $\begin{array}{lr}\text { - La RSE } & \text { se } \\
\text { rapporte } & \text { au } \\
\text { respect des droits } \\
\text { humains et des } \\
\text { normes du travail } \\
\text { ainsi qu'à la } \\
\text { conservation de } \\
\text { la nature et la } \\
\text { prévention de la } \\
\text { pollution }\end{array}$ \\
\hline 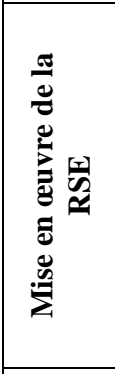 & $\begin{array}{l}\text { - L'intégration des } \\
\text { intérêts des Parties } \\
\text { Prenantes }\end{array}$ & $\begin{array}{l}\text { - Les valeurs } \\
\text { corporatives } \\
\text { dictent le } \\
\text { comportement } \\
\text { des entreprises } \\
\text { - La consultation } \\
\text { des Parties } \\
\text { Prenantes comme } \\
\text { moyen de mise en } \\
\text { œuvre } \\
\end{array}$ & $\begin{array}{lr}\text { - La mise en } \\
\text { œuvre nécessite } \\
\text { l'équilibre } & \text { des } \\
\text { pouvoirs } & \text { et } \\
\text { l'ouverture de la } \\
\text { gouvernance de } \\
\text { l'entreprise }\end{array}$ & $\begin{array}{l}\text { - L'ouverture de } \\
\text { la gouvernance } \\
\text { de l'entreprise }\end{array}$ \\
\hline 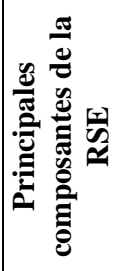 & $\begin{array}{l}\text { - La divulgation et } \\
\text { la reddition de } \\
\text { comptes }\end{array}$ & $\begin{array}{l}\text { - La consultation } \\
\text { - La reddition de } \\
\text { comptes et la } \\
\text { vérification } \\
\text { indépendante }\end{array}$ & $\begin{array}{l}\text { - La conformité } \\
\text { réglementaire } \\
\text { - La reddition de } \\
\text { comptes }\end{array}$ & $\begin{array}{l}\text { - Le respect des } \\
\text { normes } \\
\text { internationales } \\
\text { - La reddition de } \\
\text { comptes }\end{array}$ \\
\hline
\end{tabular}

\section{La reddition de comptes : un compromis au sein des différentes représentations de la RSE}

Un compromis autour de la nécessité de la reddition de comptes et de l'imputabilité de l'entreprise se dégage au sein de notre corpus. Pour les associations patronales et les entreprises, la reddition de comptes est considérée comme une 
composante essentielle de la RSE. Le succès de cette pratique socialement responsable auprès des associations patronales et des entreprises tient à de multiples raisons. Tout d'abord, la rentabilité qui prend plusieurs formes à savoir l'amélioration de l'image de l'entreprise, la motivation des employés, l'attraction des investisseurs, l'amélioration de l'avantage compétitif, etc. Ensuite, la reddition de comptes s'intègre bien au champ de la gestion de l'entreprise en s'implantant dans un domaine très sensible qui est la gestion de risques. Enfin, la reddition de comptes envers les Parties Prenantes fournit la preuve de l'engagement de l'entreprise et de sa conformité aux normes et aux standards en vigueur.

À l'instar des associations patronales et des entreprises, les syndicats et les ONG trouvent dans l'imputabilité et la reddition de comptes des entreprises un mécanisme par lequel l'entreprise se conforme aux valeurs de la société. Le respect des droits humains, du droit de travail et des normes de préservation de la nature doit faire l'objet d'un bilan grâce auquel les différentes Parties Prenantes, notamment les ONG, seront en mesure de contrôler le comportement des entreprises. Dans ce contexte, la reddition des comptes est perçue comme une force régulatrice permettant d'ouvrir la gouvernance de l'entreprise. Toutefois, le compromis qui se dessine autour de la reddition de comptes se dissipe lorsqu'il est question de rendre obligatoire cette pratique. Les associations patronales et les entreprises prônent une reddition de comptes volontaire encadrée par une norme procédurale. Par contre, pour les syndicats et les ONG, la pratique de reddition de comptes ne peut contribuer à la responsabilisation des entreprises en l'absence d'un cadre réglementaire qui la définit. Sans règlements pour encadrer la divulgation volontaire, les forces régulatrices de cette pratique ne peuvent pas se déployer parce qu'elle souffre encore de plusieurs omissions importantes et du manque de vérification.

\section{CONCLUSION}

Il n'est pas surprenant de constater que l'analyse du discours des associations patronales, des entreprises, des syndicats et des ONG contient des représentations irréconciliables à plusieurs égards, mais nous sommes néanmoins parvenus à identifier des convergences au sein de cet ensemble. La prise en compte des Parties Prenantes est certes la représentation dominante de la RSE dans le milieu des affaires. Que ce soit pour les associations patronales ou pour les entreprises, la RSE est par essence volontaire et se définit différemment d'une partie prenante à l'autre. Cette vision est soutenue en partie par les syndicats bien que ceux-ci aimeraient conjuguer obligation légale et initiatives volontaires soulignant ainsi la nécessité de trouver la meilleure équation d'une régulation hybride. Quant aux ONG, le rôle de l'État est de veiller sur le respect des droits humains et de l'environnement et à ce chapitre, elles se positionnent en faveur de l'adoption de normes sanctionnables par l'État en matière de RSE. Toutefois, notre analyse laisse penser que le compromis sur la RSE portera plus particulièrement sur les normes procédurales, c'est-à-dire la reddition de comptes et la certification, évacuant ainsi l'épineuse question du contenu de la responsabilité sociale. 
À l'issue de ces audiences publiques, la Commission sur la démocratie canadienne et la responsabilisation des entreprises a publié en 2002 un rapport de 21 recommandations, principalement axées sur la communication et la prise en compte des groupes d'intérêt. Selon la Commission, les entreprises souhaitant s'inscrire en bourse devraient produire une déclaration de principes les engageant à respecter l'environnement, les droits de la personne et les consommateurs. Aussi, une loi devrait également être adoptée pour protéger les employés dénonçant les pratiques frauduleuses de leur employeur. Par ailleurs, le gouvernement devrait accorder son aide seulement aux entreprises respectueuses des normes de responsabilité sociale. Toujours selon ce rapport, à l'instar du Québec et du Manitoba, le gouvernement fédéral et les autres gouvernements provinciaux devraient interdire que les entreprises et les syndicats participent au financement des partis politiques (Commission sur la démocratie canadienne et la responsabilisation des entreprises, 2002). Depuis la tenue de cette commission, la Loi sur les sociétés par actions a été modifiée si bien que les actionnaires peuvent dorénavant déposer des résolutions à caractère social, sans risques de les voir rejeter par la direction de l'entreprise. Cependant, le reste des recommandations sur la communication et la prise en compte des groupes d'intérêt est demeuré lettre morte.

\section{BIBLIOGRAPHIE}

Backhaus, K. B., Stone, B. A. \& Heiner, K. 2002. Exploring the relationship between corporate social performance and employer attractiveness. Business and Society, 41, (3): 292-318.

Caron, M.-A. \& Turcotte, M.-F. 2006. La métaphore de la mesure : étude du potentiel régulatoire des pratiques de divulgation en matière de développement durable. In R. Huet (dir.) Responsabilité sociale : vers une nouvelle communication des entreprises: 155-176. Villeneuve d'Ascq: Presse Universitaires du Septentrion.

Corley, K. G, Cochran, P. L. \& Comstock, T. G. 2001. Image and the impact of public affairs management on internal stakeholders. Journal of Public Affairs, 1(1): 53-66.

Epstein, E. M. 1987. The corporate Social Policy Process: Beyond Business Ethics, Corporate Social Responsibility, and Corporate Social Responsiveness. California Management Review, 29(3): 99-114.

Gendron, C. 2000. Le questionnement éthique et social de l'entreprise dans la littérature managériale. Cahiers du CRISES, Collection Working Papers-Études théoriques, $\mathrm{n}^{\circ} \mathrm{ET0004}$, février.

Greening, D. W. \& Turban, D. B. 2000. Corporate social performance as a competitive advantage in attracting a quality workforce. Business and Society, 39(3) : 254280.

Jodelet, D. 1989. Les représentations sociales. Paris: Paris Université France.

Luce, R. A. Barber, A. E. \& Hillman, A. J. 2001. Good deeds and misdeeds : A mediated model of the effect of corporate social performance on organizational attractiveness. Business and Society, 40(4): 397-415.

Revue Internationale de Psychosociologie, année 2010 
Morris, S. A. 1997. Internal Effects of Stakeholder Management Devices. Journal of Business Ethics, 16(4): 413-424.

Nadeau, J.-B. 2002. Entretien avec Claude Lamoureux : civilisons les entreprises ! Actualité, 27(18): 76.

Pasquero, J. 2005. La responsabilité sociale de l'entreprise comme objet des sciences de gestion : un regard historique. In M.-F. B. Turcotte et A. Salmon, (dir.) Responsabilité sociale et environnementale de l'entreprise: 80-111. Québec: Presse de l'université du Québec.

Riordan, C. M., Gatewood, R. D. \& Bill, J. B. 1997. Corporate image : Employee reactions and implications for managing corporate social performance. Journal of Business Ethics, 16: 401-412.

Schmidt-Albinger, H. \& Freeman, S. J. 2000. Corporate social performance and attractiveness as an employer to different job seeking populations. Journal of Business Ethics, 28: 243-253.

Simard, G. \& Tremblay, M. 2000. La mobilisation du personnel : l'art d'établir un climat d'échanges favorable basé sur la réciprocité. Gestion, 30(2): 60-68.

Sethi, P. S. 1975. Dimensions of Corporate Social Performance: An analytical Framework, California Management Review, 17(3): 58-64.

Stenzel, J. \& Stenzel, C. 2005. The payoffs for corporate social responsability : a conversation with Marc J. Epstein. Cost Management, 19(4): 5-13.

Teoh, H. Y. \& Shiuiu, G. Y. 1990. Attitudes towards corporate social responsability and perceived importance of social responsability information characteristics in a decision context. Journal of Business Ethics, 9: 71-77.

Turban, D. B. \& Cable, D. M. 2003. Firm reputation and applicant pool characteristic. Journal of Organizational Behavior, 24: 733-751.

Turban, D. B. \& Greening, D. W. 1997. Corporate social performance and organizational attractiveness to prospective employees. Academy of Management Journal, 40: 658-672.

Waddock, S. A. \& Graves, S. B. 1997. The corporate social performance-financial performance link. Strategic Management Journal, 18(4): 303-319.

Wood, D. J. 1991. Corporate Social Performance Revisited. Academy of Management Review, 16(4): 691-718. 